\title{
Catalytic Thermodynamics of Nanocluster Adsorbates from Informational Statistical Mechanics
}

\author{
Forrest H. Kaatz ${ }^{* \dagger} \quad$ Adhemar Bultheel ${ }^{\ddagger}$
}

\begin{abstract}
This Letter presents a new approach for studying the catalytic thermodynamics of cuboctahedral nanoclusters, using informational statistical mechanics. The Morse potential determines bond energies between cluster atoms in a coordination type calculation. Applied density functional theory (DFT) calculations demonstrate adatom effects on the thermodynamic quantities, which are derived from a Hamiltonian. Calculations of the entropy, free energy, and total energy show linear behavior, as the coverage of oxygen on platinum, and hydrogen on palladium, increases from bridge sites on the surface. The data exhibits size effects for the measured thermodynamic properties with cluster diameters between 2 and $5 \mathrm{~nm}$. Entropy and enthalpy calculations of $\mathrm{Pt}-\mathrm{O}_{2}$ compare well with previous theoretical data for $\mathrm{Pt}(111)-\mathrm{O}_{2}$, and trends for $\mathrm{Pd}-\mathrm{H}$ are similar to experimental measurements on $\mathrm{Pd}-\mathrm{H}_{2}$ nanoclusters. These techniques are applicable to a wide variety of cluster - adsorbate interactions, encouraging further research.
\end{abstract}

Keywords: catalysis; Shannon entropy; information theory; complex networks

\section{Introduction}

From the earliest history, thermodynamics has had ties to information theory. Maxwell illustrated the relationships between entropy and information with his demon, which apparently could violate the second law of thermodynamics [1]. Recently, there has been experimental work verifying the roles of information and thermodynamics, fluctuation, the Szilard engine, and Landauer's principle [2-4]. For recent reviews the reader is directed to references $[5,6]$. Also, the relationship between quantum thermodynamics and information is being explored, where the number of particles is small [7]. The interest in nanoscale science is emphasizing the differences between bulk and quantum effects, and in this manuscript, we will consider nanoscale calculations where many particles or atoms exist, so is not quite quantum in nature, yet not bulk either.

*Mesalands Community College, 911 South 10th Street, Tucumcari, NM, 88401, USA

${ }^{\dagger}$ Corresponding author email fhkaatz@gmail.com

${ }^{\ddagger}$ Dept. Computer Sci., KU Leuven, Celestijnenlaan 200A, 3001 Heverlee, Belgium 
Informational statistical mechanics has its foundations in a classic paper by Shannon [8], where he laid the groundwork for understanding information theory and the maximum entropy. Jaynes did further work $[9,10]$ on the physics of information theory where he describes the use of a partition function leading to the maximum entropy estimate in terms of statistical principles. More recently, Estrada and Hatano [11] provided a complex network approach to information theory based on graph theory and statistical mechanics. We view platinum nanoclusters as graphs, with nearest neighbor interactions, and derive some of the thermodynamic properties of adatoms on the cluster surfaces.

The Morse potential [12] was originally developed for the hydrogen molecule, but as numerical computing became established, soon became used in bulk cubic metals, and the properties were found in agreement with experimental elastic constants and the equation of state [13]. The Morse potential has been used in adatom studies of Pt/Pt surfaces with good results [14]. Although the Morse potential has a long history, recently it has been proven popular for determining local and global structural minima for free standing nanoclusters and binary clusters with a fixed size [15-17]. Nanocluster cubic metals have been analyzed with a Morse potential, with the result that the potential well becomes shallower for small clusters [18]. Here the more general method of Girifalco and Weizer [13,19] is applied to Morse potentials for clusters, with similar conclusions. We recognize that these methods are not robust DFT calculations, yet the application of a computationally tractable Morse potential allows us to gain insight into physical systems that are hard to model.

Platinum catalysis is used in many industrial applications, and there is widespread agreement that size and shape (surface exposure) is critical to cluster behavior. There are many scientists studying the physics, chemistry, and materials science of the catalytic behavior of nanoclusters. Recent research has developed methods of synthesis of Pt clusters with a chosen size and shape so that advanced surface studies may be considered [20]. In particular, we study the thermodynamics of oxygen radicals and oxygen molecules on platinum cuboctahedral surfaces, with both (111) and (100) surface orientations.

The palladium-hydrogen system is of interest technologically and scientifically [21]. Although there are bulk solid solution phases [22], on a clean surface hydrogen molecules $\mathrm{H}_{2}$ will dissociate into two hydrogen atoms and chemisorb onto (100) [23] and (111) [24] surfaces. This is because the energy of adsorption $(0.53 \mathrm{eV})$ is much larger than the energy of dissolution $(0.2 \mathrm{eV})$ into the bulk [25]. Hydrogen therefore occupies sites on the surface before penetrating into the bulk. It has been found that bridge and hollow sites are preferred over top sites on both the (100) [25], and (111) [26] surfaces. Palladium nanocrystals can be formed in many types of cluster shapes and sizes [27]. Recently, nanoplasmonic sensing has enabled scientists to study individual nanoparticles, rather than surfaces, films, or even ensembles of clusters [28]. Our studies provide a computational method to study the effects of nanocluster size on thermodynamic properties, and to compare computational data with experimental data. Although we study platinum and palladium clusters, our method is general and may be applied to a wide variety of nanocluster adsorbates. 


\section{Methods}

For the nanocluster, we get the energies from the Morse potential analysis

$$
\hat{H}=\hat{T}+\hat{V} \quad \text { or } \quad H=\sum_{i} \frac{p_{i}^{2}}{2 \mu}+\frac{D N}{2} \sum_{i}\left[\exp \left(-2 \alpha\left(r_{i}-r_{0}\right)\right)-2 \exp \left(-\alpha\left(r_{i}-r_{0}\right)\right)\right]
$$

where $\hat{T}$ and $\hat{V}$ are the momentum and potential energy respectively, $H$ is the Hamiltonian, $p_{i}$ is the momentum of the individual masses, $\mu$ is the reduced mass, $D$ is a constant related to the depth of the potential well, $\alpha$ is an inverse linewidth related to the curvature of the potential, $N$ is the number of atoms in the cluster, and $r_{0}$ is the location of the well minimum. The constants $D, \alpha$ and $r_{0}$ are determined by solving the following three equations [19]:

$$
E_{C}=\left.\frac{V}{N}\right|_{a_{0}}=D e^{\alpha}\left\{\frac{1}{2} e^{\alpha} \sum_{i=1}^{N} \exp \left(-2 \alpha M_{i} \frac{a_{0}}{r_{0}}\right)-\sum_{i=1}^{N} \exp \left(-\alpha M_{i} \frac{a_{0}}{r_{0}}\right)\right\}
$$

where $r_{i}=a M_{i}, E_{C}$ is the cohesive energy, and $a_{0}$ is the experimental lattice constant, at some central location of the cluster. The cuboctahedral clusters with an even number of shells, $L$, do not have an atom at a central location of the cluster, so we use clusters with an odd number of shells in our analysis. The equilibrium condition

$$
\left.\frac{\partial V}{\partial a}\right|_{a_{0}}=0=e^{\alpha} \sum_{i=1}^{N} M_{i} \exp \left(-2 \alpha M_{i} \frac{a_{0}}{r_{0}}\right)-\sum_{i=1}^{N} M_{i} \exp \left(-\alpha M_{i} \frac{a_{0}}{r_{0}}\right) .
$$

and the bulk modulus complete the equations to determine the constants.

$$
B=\left.\frac{1}{9 c N a} \frac{\partial^{2} V}{\partial a^{2}}\right|_{a_{0}}=\frac{D \alpha^{2} e^{\alpha}}{9 c r_{0} a_{0}}\left\{2 e^{\alpha} \sum_{i=1}^{N} M_{i}^{2} \exp \left(-2 \alpha M_{i} \frac{a_{0}}{r_{0}}\right)-\sum_{i=1}^{N} M_{i}^{2} \exp \left(-\alpha M_{i} \frac{a_{0}}{r_{0}}\right)\right\}
$$

where $c=1 / 4$ for fcc crystals.

For nanoclusters the bulk modulus varies as [29]

$$
\frac{B\left(D_{e}\right)}{B_{b u l k}}=\frac{a_{0}}{a\left(D_{e}\right)}\left(\frac{\Theta\left(D_{e}\right)}{\Theta_{B}}\right)^{2}, \quad a\left(D_{e}\right)=a_{0}-\frac{a_{0}}{1+\frac{G D_{e}}{\gamma_{s v}\left(D_{e}\right)}}, \quad \gamma_{s v}\left(D_{e}\right) \approx \gamma_{s v 0}\left(1-4 D_{a} / D_{e}\right)
$$

where $G$ is the shear modulus and $\gamma_{s v 0}$ is the surface energy, $D_{e}$ the effective nanocluster diameter, and $D_{a}$ is the atomic diameter. In Table 1 we list constants for the analysis of the equations for platinum and palladium.

The cohesive energy is [33]

$$
E_{C}=E_{B} \frac{\overline{c n}_{N}}{\overline{c n}_{B}} \text { where } \overline{c n}_{N}=\frac{1}{N} \sum_{i=1}^{N} c n_{i} .
$$




\begin{tabular}{|c|c|}
\hline $\mathrm{Pt}$ & $\mathrm{Pd}$ \\
\hline$E_{B, P t}=-5.84 \mathrm{eV}[30]$ & $E_{B, P d}=-3.89 \mathrm{eV}[30]$ \\
\hline$G_{P t}=60.9 \mathrm{GPa}[31]$ & $G_{P d}=43.5 \mathrm{GPa}[31]$ \\
\hline$d_{a}=0.26 \mathrm{~nm}[31]$ & $d_{a}=0.256 \mathrm{~nm}[31]$ \\
\hline$a_{0}=0.39233 \mathrm{~nm}[31]$ & $a_{0}=0.38901 \mathrm{~nm}[31]$ \\
\hline$B_{P t}=230 \mathrm{GPa}[31]$ & $B_{P d}=180 \mathrm{GPa}[31]$ \\
\hline$\gamma_{s v 0}=2.85 \mathrm{~J} / \mathrm{m}^{2}[32]$ & $\gamma_{s v 0}=2.35 \mathrm{~J} / \mathrm{m}^{2}[32]$ \\
\hline
\end{tabular}

Table 1: Constants for the derived parameters of equations 2, 3, and 4, for platinum and palladium.

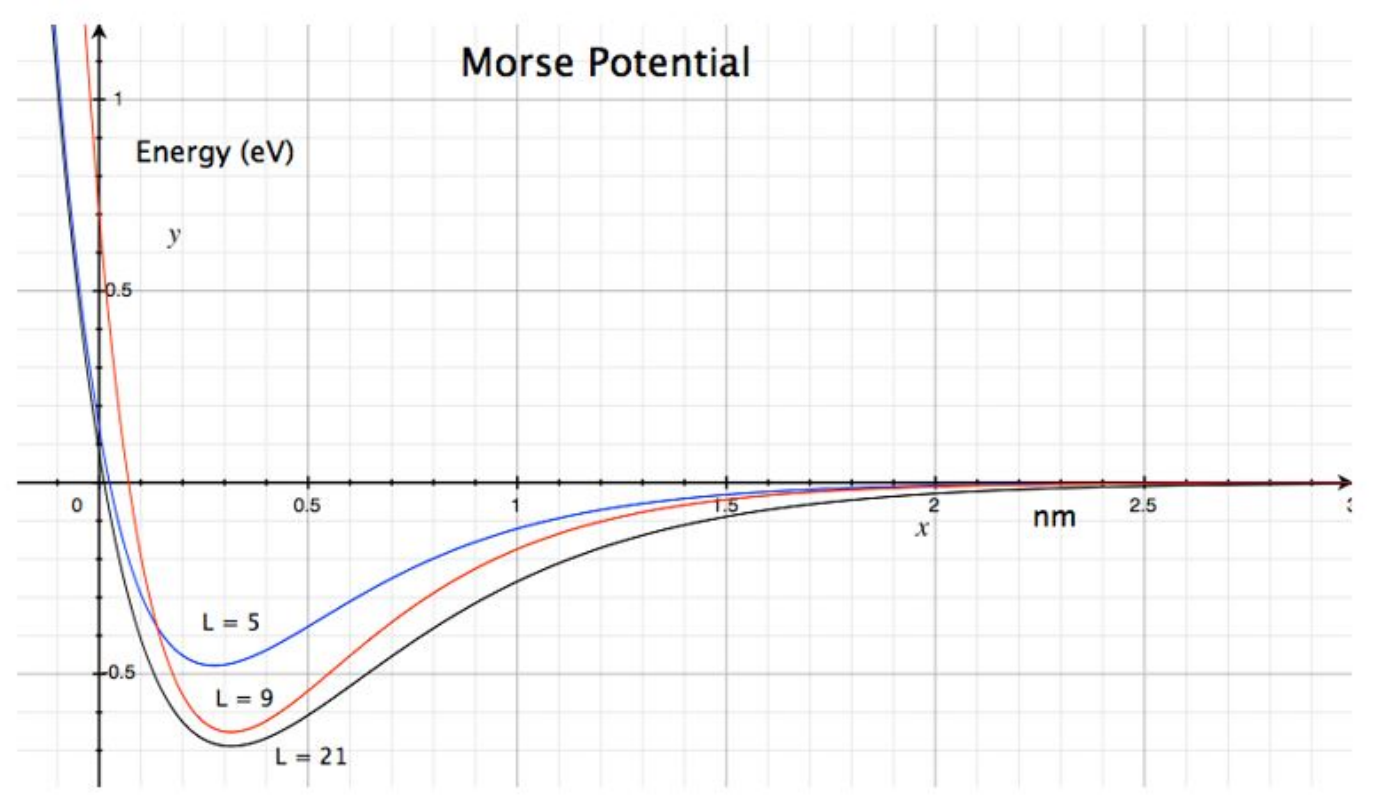

Figure 1: Plots of two-body Morse potential with different cluster sizes for platinum. 


\begin{tabular}{|c|c|c|c|c|c|c|c|}
\hline \multirow{2}{*}{$\begin{array}{c}\text { System } \\
\text { Parameters }\end{array}$} & \multicolumn{6}{|c|}{$L$} \\
\cline { 3 - 8 } & 5 & 7 & 9 & 11 & 13 & 21 \\
\hline $\mathrm{Pt}$ & $D(\mathrm{eV})$ & 0.47781 & 0.632562 & 0.6520041 & 0.663494 & 0.677404 & 0.688523 \\
\hline $\mathrm{Pt}$ & $\alpha\left(\mathrm{nm}^{-1}\right)$ & 2.76046 & 2.83172 & 2.832589 & 2.832412 & 2.832105 & 2.831845 \\
\hline $\mathrm{Pt}$ & $r_{0}(\mathrm{~nm})$ & 0.275916 & 0.31586 & 0.315433 & 0.316672 & 0.315799 & 0.315992 \\
\hline $\mathrm{Pt}$ & diam $(\mathrm{nm})$ & 1.96 & 2.75 & 3.53 & 4.32 & 5.10 & 8.24 \\
\hline \hline $\mathrm{Pd}$ & $D(\mathrm{eV})$ & 0.41005 & 0.44505 & 0.45791 & 0.46585 & 0.47139 & 0.48319 \\
\hline $\mathrm{Pd}$ & $\alpha\left(\mathrm{nm}^{-1}\right)$ & 2.83889 & 2.85412 & 2.85429 & 2.85395 & 2.85368 & 2.85308 \\
\hline $\mathrm{Pd}$ & $r_{0}(\mathrm{~nm})$ & 0.305010 & 0.29918 & 0.29917 & 0.29936 & 0.29950 & 0.29983 \\
\hline $\mathrm{Pd}$ & diam $(\mathrm{nm})$ & 1.95 & 2.72 & 3.50 & 4.28 & 5.06 & 8.17 \\
\hline \hline & $N($ atoms $)$ & 490 & 1,288 & 2,670 & 4,796 & 7,826 & 32,186 \\
\hline
\end{tabular}

Table 2: Fitted values of the parameters from MATLAB analysis.

$E_{B}$ is the cohesive energy of the bulk $(\approx-3$ to $-6 \mathrm{eV})$ and $\overline{c n}_{B}=12$ for an fcc crystal. This data is linear with $N^{-1 / 3}$ so that the cohesive energy is related to the surface area of the clusters, as described in a review article [34].

The Debye temperature is given by [33]:

$$
\Theta\left(D_{e}\right)=\Theta_{B} \sqrt{E_{C} / E_{B}}
$$

so that equation (5) is directly related to the cohesive energy.

The eigenvalues of the Hamiltonian in the two-body case in the ground state to first order are [12]

$$
E_{0} \approx-D+\frac{h \omega_{0}}{2}, \quad \omega_{0}=\frac{\alpha}{2 \pi} \sqrt{\frac{2 D}{\mu}}, \quad \mu=\frac{M_{1} M_{2}}{M_{1}+M_{2}}
$$

where $h$ is Plank's constant, and $M_{1}$ and $M_{2}$ are the two-body masses.

The changes in the Morse potential with cluster size is shown in Figure 1, and the data is listed in Table 2 up to $L=21$ shells in the cluster. The potential is shallower as the cluster becomes smaller, and the minimum well position shifts to smaller $r_{0}$ as well. There is a crossover in the repulsive part of the potential from $L=5$ to $L=9$, which may be elementally specific. These effects are in agreement with a recent publication [18].

In the case of $n$-fold bonding, the energies become [35]

$$
E_{c n}=E_{0}(2-1 / c n) \quad \text { or } \quad E\left(c n_{i}\right) \approx\left(-D+\frac{h \omega_{0}}{2}\right)\left(2-\frac{1}{c n_{i}}\right)
$$

where $c n_{i}$ is the number of bonds or the coordination of the $i$ th site. Figure 2 shows how the coordination affects the bond energy in the platinum clusters, as determined by equation (9). These coordination-style calculations, show clear evidence of a size effect, primarily 


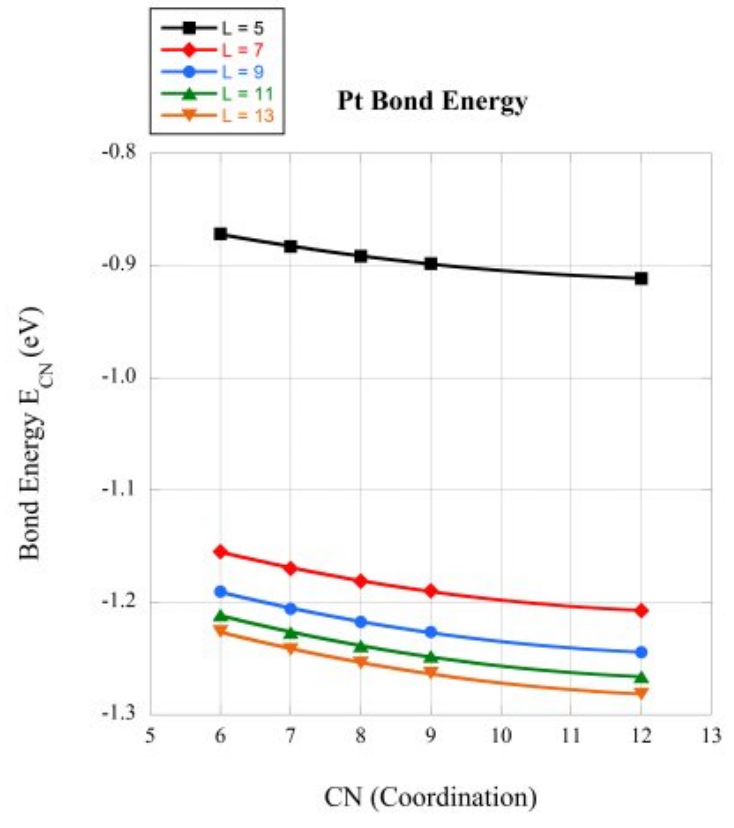

A

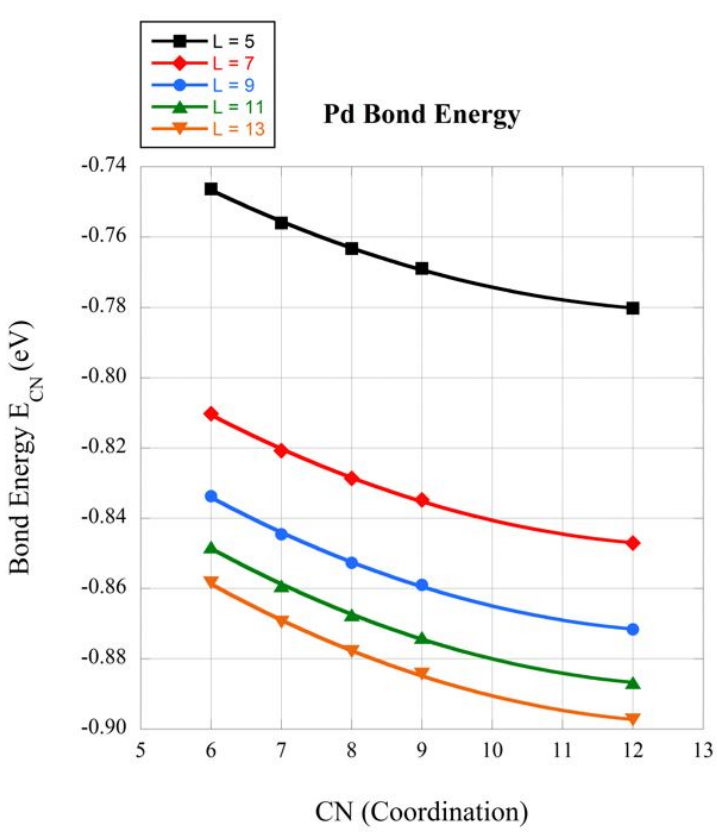

$\mathrm{B}$

Figure 2: Platinum (A) and palladium (B) cluster bond energies as the coordinates changes. 


\begin{tabular}{|c|c|c|}
\hline species & offset vs $\overline{C N}(\mathrm{eV})$ & slope vs $\overline{C N}$ \\
\hline${ }^{*} \mathrm{O}$ & -1.936 & 0.118 \\
\hline${ }^{*} \mathrm{O}_{2}$ & -2.418 & 0.227 \\
\hline
\end{tabular}

Table 3: Linear fits from density functional theory based on a coordination model [37].

due to the constant $D$, which is tabulated in Table 2. Also, the bonding is stronger as $c n$ increases, in line with what we might expect.

Adsorbates on cuboctahedral clusters may sit on a bridge site, a 3 hollow on (111) surfaces or a 4 hollow on (100) surfaces. Figure 3 shows figures of some random possibilities for collections of adsorbates on the four sizes of clusters we examine, with $L=5,7,9,11$. We recognize that edge and corner sites are relevant in the adsorption of gasses on clusters, but it is difficult to assign the coverage of these sites to any particular surface geometry. Therefore only the facets are considered when calculating the surface coverage, where we have well-defined (111) or (100) surfaces. Adatom coverage is measured as $\theta=N_{a} / N_{s}$ where $N_{a}$ is the number of adsorption sites, and $N_{s}$ is the number of surface sites. From Figure 5 of reference [36], we estimate that the fraction of facet sites for platinum cuboctahedra varies from $10 \%$ to $60 \%$ as the size of the cluster increases from $1.5 \mathrm{~nm}$ to $5 \mathrm{~nm}$.

We adapt a density functional theory (DFT) for energies of adsorbates from a coordination model $[37,38]$ :

$$
\overline{C N}_{i}=\sum_{j=1}^{n_{i}} \frac{c n_{j}}{c n_{\max }}
$$

where $c n_{\max }=18$ for bridge sites, 22 for 3 hollows and 26 for 4 hollows [38]. Adsorbate energy is linear with coordination:

$$
E_{A B}=\overline{C N}_{0}+m \overline{C N}
$$

Table 3 shows the values of the linear fits as determined by reference [37]. The bond energy bond order (BEBO) model describes bond energy with bond order, $n$, as [39]

$$
E_{A B}=E_{a} c n_{A B}^{p}
$$

where $E_{A B}$ is the adsorption bond energy, $c n$ is the bond order, and $p$ an exponent which is normally unity. The BEBO model has been used for $\mathrm{O}_{2}$ adsorbates on $\mathrm{Pt}$ (111) [39]. In practice, this results in dividing the adsorption energy by the number of bonds in the surface site [39].

Likewise, for the Pd-H system, a Morse potential models the surface hydrogen-palladium interaction [40]. These parameters are listed in Table 4. For bridge sites, we adjust the bonding by a factor of two, for the bridge bond as in equation (12). The bonding can also be adjusted for 3 hollow and 4 hollow sites on the surfaces. The Morse potential was successfully used to model $\mathrm{Pd}-\mathrm{H}$ nanoclusters and determine the adsorption energies and bond lengths of adsorbed hydrogen in different sites [40]. 

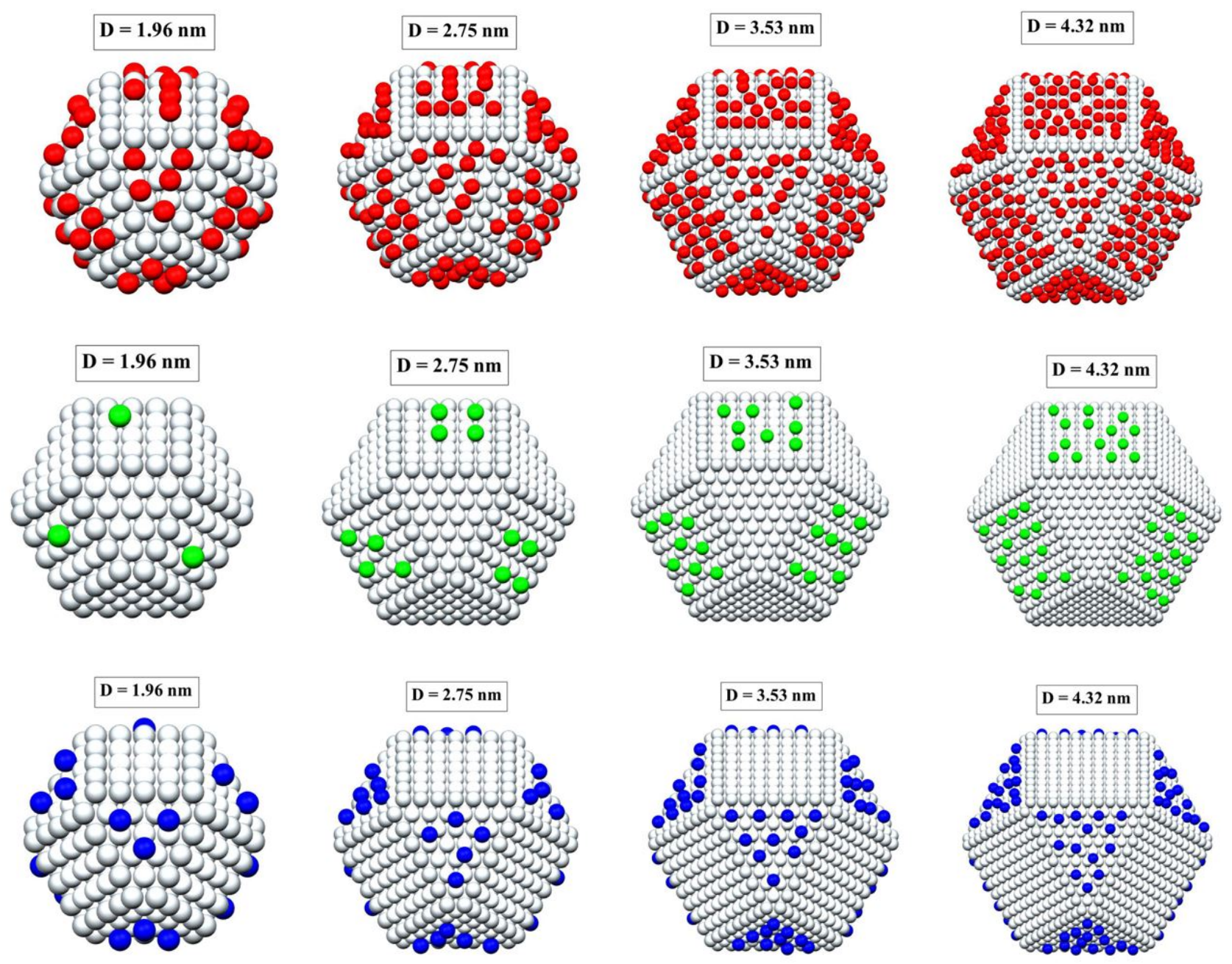

Figure 3: Platinum clusters with adatoms in bridge, 4 hollows (100), and 3 hollow (111) surfaces. 
The adsorption energies critically influence the thermodynamic properties. Some indication of the importance of surfaces and adsorption is shown in Figure 4 for $\mathrm{Pt}^{*} \mathrm{O}$ nanoclusters. The surface energy, $\gamma_{s v}\left(D_{e}\right)$ is obtained from [41].

$$
\gamma_{s v}\left(D_{e}\right)=\gamma_{s v 0}\left[1-\frac{1}{2 D_{e} / D_{a}-1}\right] \exp \left(-\frac{2 S_{B}}{3 R} \frac{1}{2 D_{e} / D_{a}-1}\right)
$$

where $S_{B}=124.34 \mathrm{~J} / \mathrm{mol}-\mathrm{K}$ is the entropy of the solid-vapor transition and $R$ is the gas constant. The adsorption energy, $E_{a}$, is taken from equations (11) \& (12), where we use bridge sites, at full coverage, for 6,7,8 and 9 coordinated atoms and divide the sum by the total number of surface atoms. These two quantities are plotted versus platinum nanocluster diameter. We find $E_{a}$ to be $\approx-0.48 \mathrm{eV}$, as the size gets large, while the experimental value for bulk $\mathrm{Pt}(111)-\mathrm{O}^{*}$ surfaces is about $-0.35 \mathrm{eV}$ [42]. Our calculated value includes corners and edges, which are lower coordinated, thus make a larger contribution to the adsorption energy than one would have from facets on the (111) surface. Moreover, DFT calculations of $\mathrm{Pt}$ clusters show that the oxygen adsorption energy becomes approximately constant for sizes greater than about $2 \mathrm{~nm}$, although only bridge sites on edges were considered in the DFT calculations [43]. The plot of $\left.\gamma_{s v}\left(D_{e}\right)\right)$ versus platinum nanocluster diametea in Figure 4 nears the value $2.5 \mathrm{~J} / \mathrm{m}^{2}$ for $D_{e}=9 \mathrm{~nm}$ and slowly approaches the the bulk value of $2.85 \mathrm{~J} / \mathrm{m}^{2}$, as the diameter becomes large (not shown). Note that the value $2.85 \mathrm{~J} / \mathrm{m}^{2}$ is an average over the (111) and (100) surfaces for the cuboctahedral geometry [32].

To allow for small deviations from the average bond length, we define $i$ and $j$ as nearest neighbors, and separate them from the rest by requiring that $r_{i j}<r_{c}$ where $r_{c}$ is a threshold value, appropriate for the nanocluster. Thus,

$$
A(i, j)=\left\{\begin{array}{l}
1 \text { if } r_{i j}<r_{c} \text { and } i \neq j \\
0 \text { otherwise }
\end{array}\right.
$$

describes the adjacency matrix for the cluster. The approximate energies for bonds in the cluster and the adatoms are:

$$
\begin{gathered}
E(i, j)=\left[E\left(c n_{i}\right)+E\left(c n_{j}\right)\right] / 2 \\
\mathbf{H}(i, j) \approx \begin{cases}E(i, j), & \text { if } i \text { and } j \text { are in the nanocluster } \\
E_{a}(i, j), & \text { if } i \text { or } j \text { is an adatom } \\
0 & \text { otherwise }\end{cases}
\end{gathered}
$$

with only nearest neighbor non-zero entries, i.e. a sparse matrix.

In the Hamiltonian approach, the partition function is $[9,11]$

$$
Z=\operatorname{Tr}\left(e^{-\beta \mathbf{H}}\right)
$$

and $\beta=1 /\left(k_{B} T\right)$ is the inverse temperature, where $k_{B}$ is Boltzmann's constant, $T$ is the temperature in Kelvin, and $\mathbf{H}$ is the Hamiltonian matrix as given in equation (16). The probability is related to the partition function as: $p_{j}=\frac{e^{\beta \lambda_{j}}}{Z}$ where $\lambda_{j}$ is an eigenvalue of 


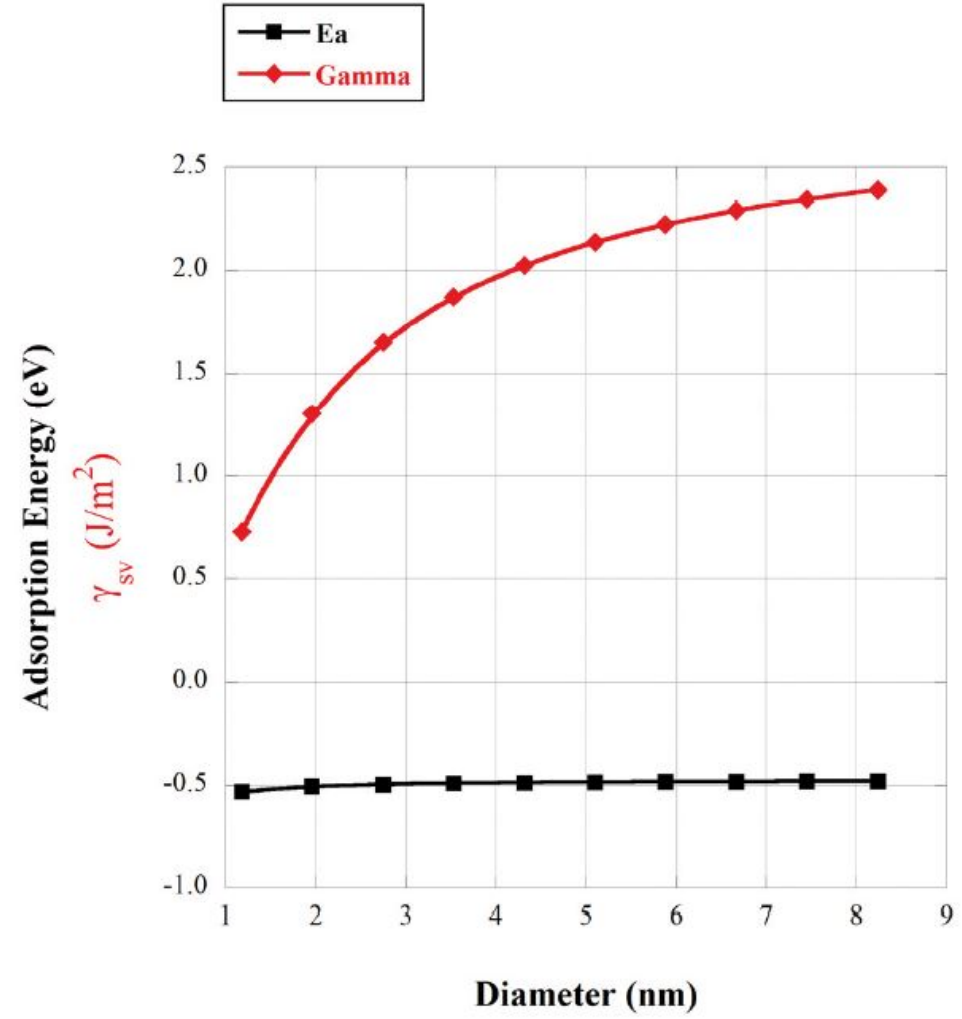

Figure 4: Calculated surface energy, $\gamma_{s v}$, and adsorption energy, $E_{a}$, versus platinum nanocluster diameter.

\begin{tabular}{|c|c|c|c|}
\hline system & $D(\mathrm{eV})$ & $\alpha\left(\mathrm{nm}^{-1}\right)$ & $r_{0}(\mathrm{~nm})$ \\
\hline Pd-H & 0.49 & 0.110 & 0.035 \\
\hline
\end{tabular}

Table 4: Constants for the Pd-H Morse potential system [40]. 
the Hamiltonian matrix. The informational Shannon entropy is then (with units of eV/K) $[8,9,44]$

$$
S=-N_{T} k_{B} \sum_{j=1}^{N} p_{j} \ln p_{j}
$$

where $N_{T}=N_{c}+N_{a}$ with $N_{c}$ the number of atoms in the cluster and $N_{a}$ the number of adatoms at coverage $\theta$. The free energy in the nanoscale systems we examine is in $\mathrm{eV}[9,11]$

$$
F=-k_{B} T \ln Z
$$

where $Z$ is the partition function, equation (6). The total energy is also in eV

$$
U=F+T S .
$$

The effect of coverage and temperature of the thermodynamic functions is measured as

$$
\Delta T_{L, \theta, T}=T_{L}(\theta, T)-T_{L}(0, T)
$$

where $T_{L}$ is the thermodynamic function value (free energy, entropy, or total energy) of the cluster with $L$ shells.

The clusters have random collections of adatoms on the surface, and particularly for smaller clusters, there is scatter in the data, so we average over 50 runs for $L=5,5$ runs for $L=7$, one run each when $L=9,11,13$ for bridge sites, and 500 runs for $L=5,20$ runs for $L=7$, for hollow sites. A custom MATLAB code calculates the above quantities. The adatoms are added sequentially in random sites until maximum coverage is reached, and equations (18), (19), and (20) are determined in the manner described.

\section{Results and Discussion}

As a reminder, the relationship between the thermodynamic variables is $F=U-T S$, $H=U+P V, G=F+P V$, where $H$ is the enthalpy, $P$ is the pressure, $V$ is the volume, and $G$ is the Gibbs energy. The enthalpy and entropy are more commonly derived experimental quantities. This comes about because of the Van't Hoff relationship:

$$
\ln \left(\frac{P}{P_{0}}\right)=\frac{\Delta H}{R T}-\frac{\Delta S}{R}
$$

where $P_{0}$ is the intercept of $\ln \left(P / P_{0}\right)$ with $1 / T$, and $R$ is the gas constant [45]. Here we assume that the $P V$ term is small to first order, so that $H \sim U$ and $G \sim F$ [46]. New methods of measuring the thermodynamics of nanoclusters may lead to sufficient accuracy to test the current model [28]. Our calculations are for freestanding clusters, whereas it has been shown that a substrate for supported platinum clusters can markedly affect the catalytic properties [47].

The temperature evolution of oxygen adsorbates on platinum clusters has been found experimentally to begin desorbing at around $400 \mathrm{~K}$, for clusters of about $5 \mathrm{~nm}$ in size [48]. 


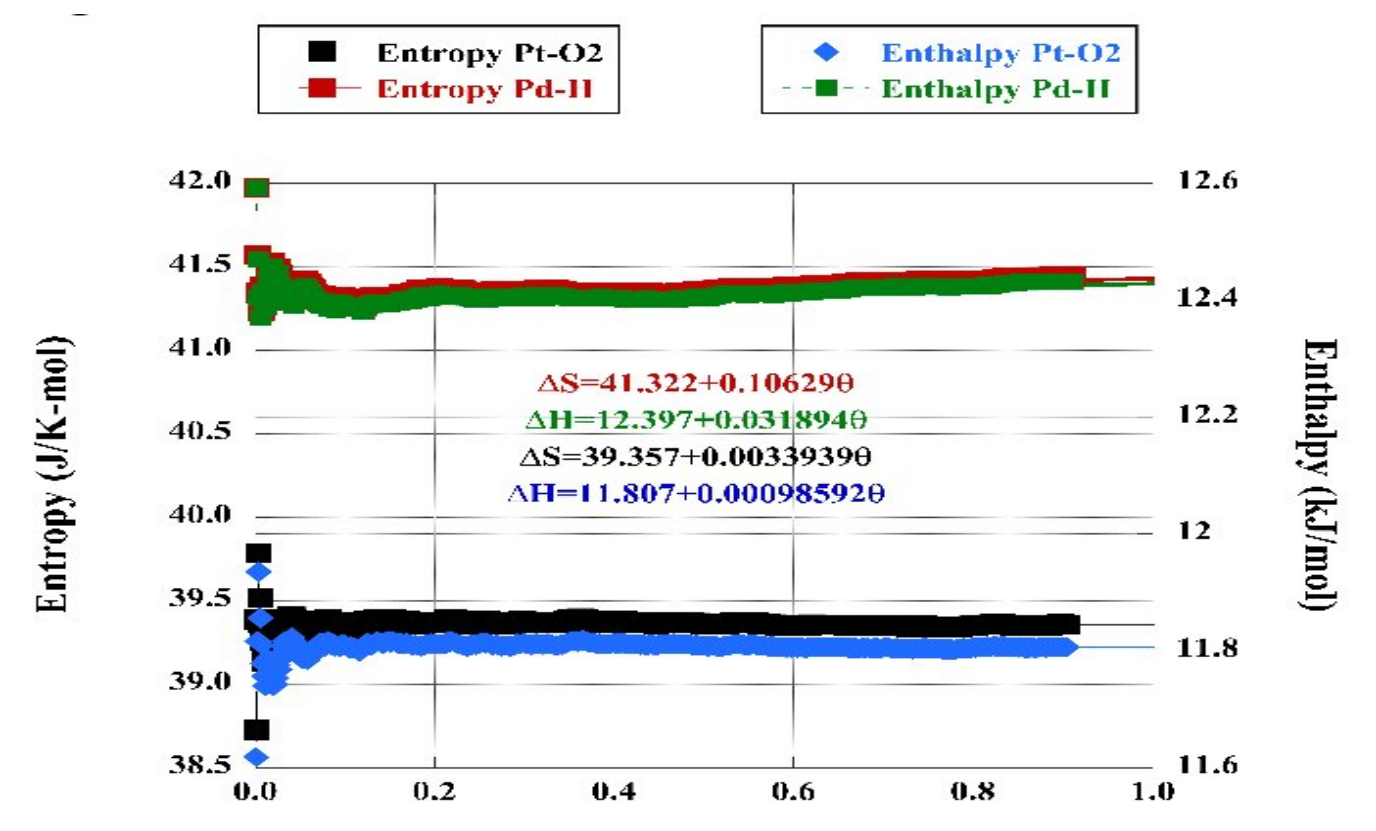

Figure 5: Linear relationships of enthalpy and entropy with coverage.

This desorption is size dependent, with smaller clusters releasing oxygen at higher temperatures. These temperatures are significantly higher than the bulk behavior, where oxygen begins desorbing at 320K from $\mathrm{Pt}$ (100) [49]. More recent studies of the oxidation of $\mathrm{Pt}$ nanoclusters on various substrates, reveal a coexistence of $\mathrm{Pt}-\mathrm{O}_{2}$ and $\mathrm{Pt}-\mathrm{O}^{*}$ with primarily Pt- $\mathrm{O}_{2}$ adsorbates until the temperature reaches $T=350 \mathrm{~K}$, and with primarily Pt-O* until $T=600 \mathrm{~K}[50,51]$. There is some variation depending on the substrate material. Platinum nanoparticles in these studies were $1.5-3.3 \mathrm{~nm}$ in diameter. This temperature effect is modeled by varying $T$ from $100 \mathrm{~K}$ to $600 \mathrm{~K}$ through the inverse temperature parameter in the partition function, at temperatures where we expect most of the oxygen in some form to remain adsorbed to the clusters.

The data exhibits linear trends with coverage, we make the ansatz $[52,53]$ :

$$
U(\theta)=U_{0}+\theta U_{1}
$$

and we extend the model to include the entropy;

$$
S(\theta)=S_{0}+\theta S_{1}
$$

where the subscript zero refers to intercepts and one to the slope. Here we model enthalpy and entropy as mass quantities, and the functions are linear with the coverage $\theta$.

Figure 5 plots the enthalpy and entropy (mass quantities) versus the coverage $\theta$ at $T=$ $300 \mathrm{~K}$, and shows linear relationships for the $5 \mathrm{~nm}$ clusters. For Platinum, a discussion in reference [54] mentions that the enthalpy is observed to be linear with oxygen absorption for 


\begin{tabular}{|c|c|c|c|c|c|}
\hline$L$ & $N_{c}$ & $\begin{array}{c}\left.N_{a} \text { (bridge }\right) \\
\text { Fig. } 3\end{array}$ & $\begin{array}{c}N_{a}(3 \text { hollow }) \\
\text { Fig. } 3\end{array}$ & $\begin{array}{c}N_{a}(4 \text { hollow }) \\
\text { Fig. } 3\end{array}$ & $\begin{array}{c}\left.N_{a} \text { (bridge }\right) \\
\text { Fig. } 6\end{array}$ \\
\hline 5 & 490 & 55 & 17 & 6 & 102 \\
\hline 7 & 1,288 & 141 & 38 & 24 & 206 \\
\hline 9 & 2,670 & 265 & 73 & 52 & 325 \\
\hline 11 & 4,796 & 420 & 111 & 87 & 541 \\
\hline 13 & 7,826 & 614 & 162 & 136 & 748 \\
\hline
\end{tabular}

Table 5: Average values of adatoms $N_{a}$, on clusters with $N_{c}$ atoms for Figures 3 and 6 .
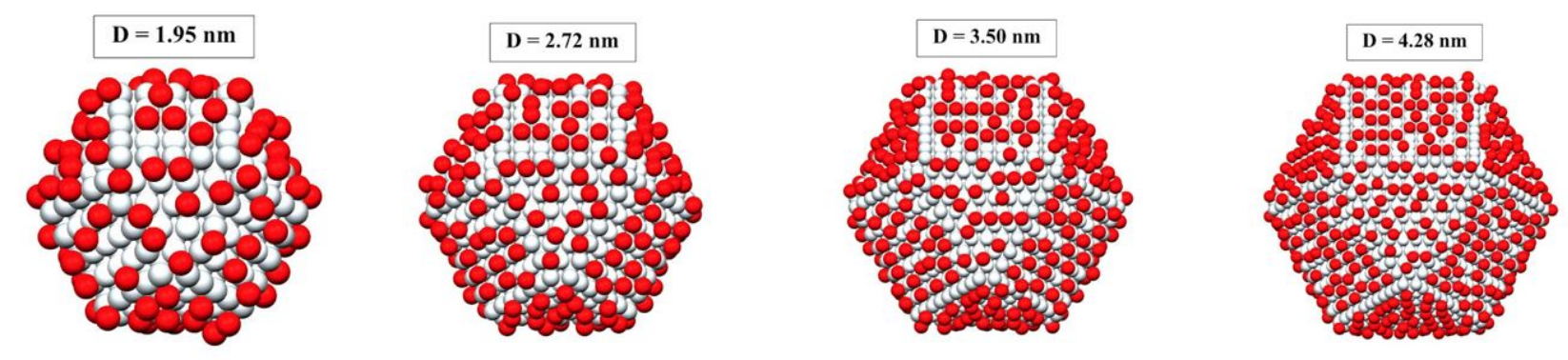

Figure 6: Palladium nanoclusters with hydrogen adsorbates over the whole cluster in bridge sites. 
$\theta<0.15$. We are not able to model desorption of oxygen at higher coverage, as is observed experimentally.

The entropy and total energy as given by equations (18) and (20) both increase quadratically with cluster size at a given temperature, while the free energy also increases, meaning it becomes less negative, in agreement with equation (18). At $350 \mathrm{~K}$ we changed the absorbate from $\mathrm{O}_{2}$ to $\mathrm{O}^{*}$ in agreement with experimental results, but the major changes in the data are the result of cluster size and temperature. Table 5 lists the average number of adatoms for each type of site. The number of adatoms increases approximately in a quadratic fashion with cluster size for each type of site at maximum coverage. Because the number of adatoms has this relationship, it is not surprising that the entropy and total energy also increase quadratically.

In Figure 6 the code is changed to allow bridge sites over the whole palladium cluster. We use bridge sites, since the cuboctahedral clusters have (100) and (111) surfaces, which hollow sites cannot completely cover. The hydrogen adsorbates fill the entire surface of the slightly smaller palladium (as compared to platinum) clusters. The average number of adatoms is listed in the last column of Table 5. The data show calculated data for $T=300 \mathrm{~K}$ of the entropy for $\mathrm{Pd}-\mathrm{H}$ clusters along with experimental results for $\mathrm{Pd}-\mathrm{H}_{2}$ from various references in Figure 7A. There is some scatter in the experimental data, which is modeled with linear regression. Our model for the size dependence is as follows:

$$
T\left(D_{e}\right)=a+b \ln \left(1-c /\left(D_{e}+d\right)\right)
$$

where $T\left(D_{e}\right)$ is the thermodynamic property, enthalpy or entropy, $D_{e}$ is the effective diameter of the clusters, and $a, b, c$, and $d$ are constants of the fit. These constants are listed in Figure 7 for the various curves.

An alternate model with fewer fit parameters is:

$$
T_{e}\left(D_{e}\right)=a \exp \left(-b D_{e}\right)+c
$$

where $T_{e}\left(D_{e}\right)$ is the exponential fit to the thermodynamic data. The fit parameters to the corresponding data in Figure 7 is shown in Table $6 . D_{0}$ is the $x$-intercept of the entropy and enthalpy models for equation 26. Also in Table $6 D_{0}^{*}$ is the corresponding $x$-intercept of equation (25). Table 6 shows that while $T_{e}\left(D_{e}\right)$ is comparable in both models, the $x$-intercept is more realistic for equation (25) than equation (26). We therefore choose equation (25) and show the respective fit to the data in Figure 7. Our fit of the averaged experimental data in Figure 7A leaves out the data from Wadell [58], as it is a possible outlier to the rest of the data. The averaged experimental data is in reasonable agreement with the calculated model, but additional work needs to done for $D_{e}<20 \mathrm{~nm}$. It is known that hydrides form more readily in nanoclusters than in the bulk [59], which may affect the measurements for small dimensions. We agree that there is little size dependence, as the diameter gets large. This model does not really reconstruct adsorption or desorption, so we do not state a preference for either type of data.

Figure 7B, shows the enthalpy model and data for the palladium-hydrogen system. Again, there is quite some scatter in the experimental data. The model follows the data 


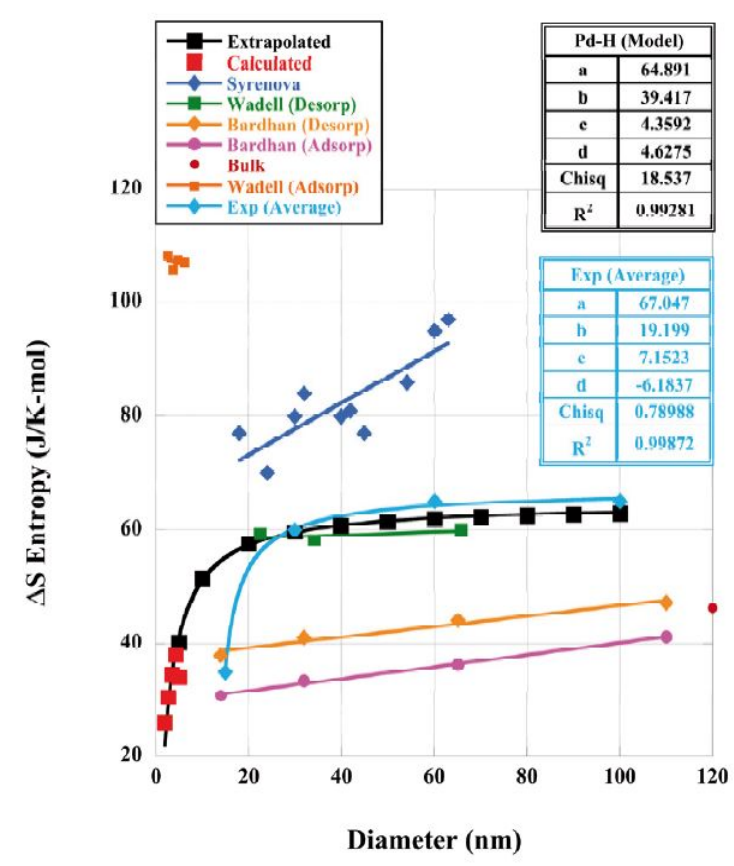

A

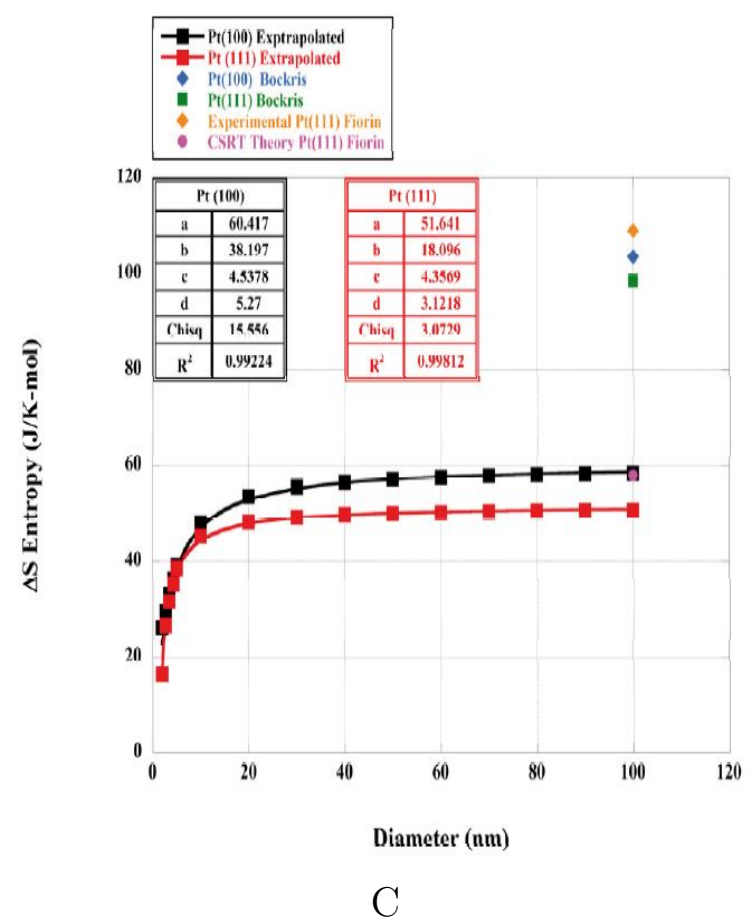

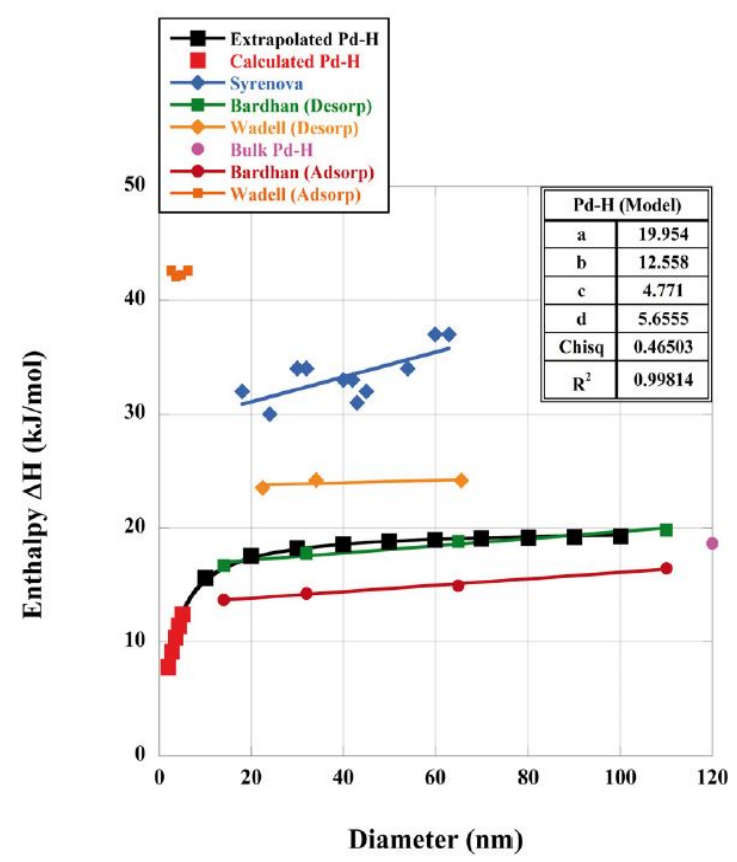

$\mathrm{B}$

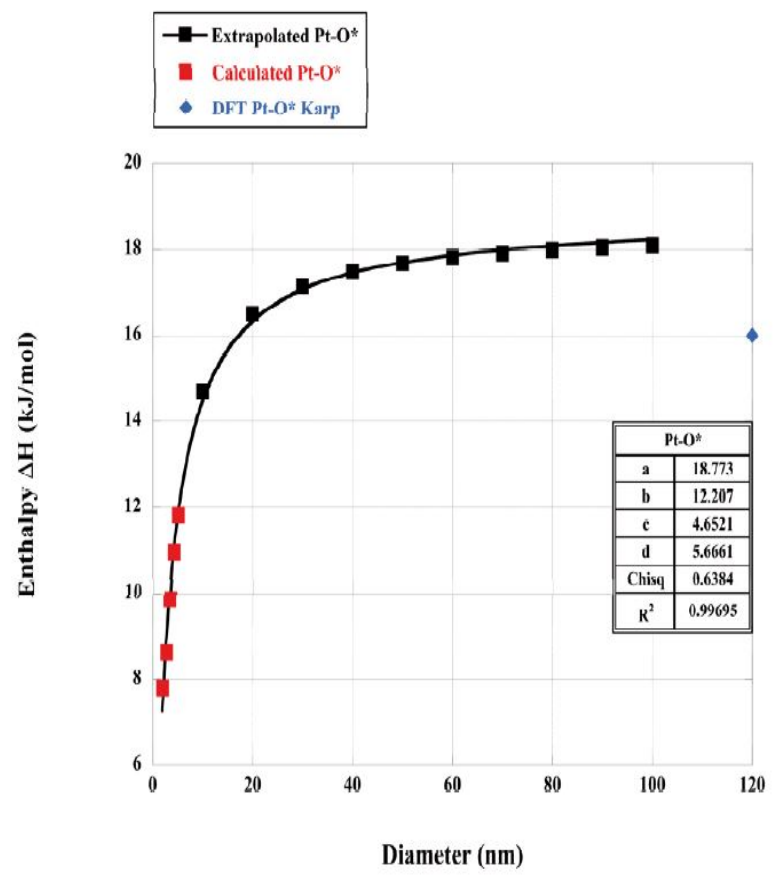

$\mathrm{D}$

Figure 7: A. Experimental and calculated entropy for $\mathrm{Pd}-\mathrm{H} 2$ and $\mathrm{Pd}-\mathrm{H}$, respectively, as a function of diameter. Data from Syrenova is from [21], Wadell (Desorp) from [55], Bardhan from [56], Bulk from [57], and Wadell (Adsorp) from [58]. B. Enthalpy for Pd-H clusters as a function of diameter. Experimental values and references as mentioned in A. C. Entropy of $\mathrm{Pt}(100)-\mathrm{O}_{2}$ and $\mathrm{Pt}(111)-\mathrm{O}_{2}$ as a function of diameter. The reference from Bockris is [60] and Fiorin from [61]. D. The calculated enthalpy data from Pt-O* is in reasonable agreement with the bulk data from Karp [54]. 


\begin{tabular}{|c|c|c|c|c|c|}
\hline Eq. (26) & $\begin{array}{c}\text { Fig. 7A } \\
\text { Pd-H(Entropy) }\end{array}$ & $\begin{array}{c}\text { Fig. 7B } \\
\text { Pd-H (enthalpy) }\end{array}$ & $\begin{array}{c}\text { Fig. 7C } \\
\mathrm{Pt}(111)\end{array}$ & $\begin{array}{c}\text { Fig. 7C } \\
\mathrm{Pt}(100)\end{array}$ & $\begin{array}{c}\text { Fig. 7D } \\
\text { Pt-O* }\end{array}$ \\
\hline$a$ & -46.317 & -14.492 & -62.832 & -41.259 & -13.21 \\
\hline$b$ & 0.1476 & 0.14919 & 0.34091 & 0.14964 & 0.14408 \\
\hline$c$ & 61.718 & 18.889 & 49.725 & 57.356 & 17.767 \\
\hline$R^{2}$ & 0.99508 & 0.99489 & 0.99153 & 0.99469 & 0.99476 \\
\hline$D_{0}(\mathrm{~nm})$ & -1.944 & -1.776 & 0.686 & 2.201 & 2.104 \\
\hline$D_{0}^{*}(\mathrm{~nm})$ & 0.464 & 0.34 & 1.502 & 0.442 & 0.259 \\
\hline
\end{tabular}

Table 6: Fit parameters and data for Figure 7 from equation (26).

from Bardhan (Desorption) [56] almost exactly. The model appears near the center of the average for the experimental data, and there is little size dependence as the clusters get large. For our model, this is the result of a horizontal asymptote in the quadratic dependences of the adatoms and thermodynamic property with size. The fit to the model is shown in the inset.

Some experimental data for platinum of the thermodynamic functions we are calculating has been published in 2009 [61], and gives a measurement for the entropy of $\mathrm{Pt}(111)-\mathrm{O}_{2}$ on bulk surfaces as $109 \mathrm{~J} / \mathrm{K}$-mol or $1.14 \times 10^{-3} \mathrm{eV} / \mathrm{K}$-particle. However, a more recent work discusses these results and the reported enthalpy was about $40 \%$ too high, due to errors in the reflectivity of platinum reported in the literature [54]. Our calculations and extrapolated enthalpy for $T=300 \mathrm{~K}$ are shown in Figure $7 \mathrm{C}$ and $7 \mathrm{D}$. The extrapolated data gives a value of $18.7 \mathrm{~kJ} / \mathrm{mol}$ as the diameter gets large. A DFT calculation in reference [51] gives a bulk value at full $\mathrm{O}^{*}$ coverage of $16 \mathrm{~kJ} / \mathrm{mol}$. Figure $7 \mathrm{C}$ shows the entropy of $\mathrm{Pt}(111)-\mathrm{O}_{2}$ and $\mathrm{Pt}(100)-\mathrm{O}_{2}$, as a function of diameter. The extrapolated values are near $60 \mathrm{~J} / \mathrm{K}$-mol. Fiorin [61] refers to the desorption entropy and compares the experimental value with one from conventional transition state theory (CTST), which varies from 40-76 J/K-mol or 58 \pm 18 $\mathrm{J} / \mathrm{K}$-mol. A previous paper in 1998 [60] gives values for the entropy for $\mathrm{Pt}(111)-\mathrm{O}_{2}$, which are close to the unadjusted value, which is probably not correct. Our results are close to the theoretical values from CTST, and if the entropy should have been reported as a lower value, as was the enthalpy, it would be close to the experimental value as well.

A basic relationship from thermodynamics is that [62]:

$$
\Delta H=\Delta S T_{\text {comp }}+\Delta G
$$

where $T_{\text {comp }}$ is the compensation temperature, $S$ is the slope and $G$ the offset. We plot this data for our model of $\mathrm{Pd}-\mathrm{H}, \mathrm{Pt}-\mathrm{O}^{*}$, and some experimental data for $\mathrm{Pd}-\mathrm{H}_{2}$ in Figure 8. Our models show good linearity, along with the $\mathrm{Pd}-\mathrm{H}_{2}$ data for desorption and adsorption. The data from Syrenova [21] for adsorption shows the most non-linearity. Note that the adsorption compensation temperature is different than that reported in [62] since not all the same data was used. The compensation temperature is related to a compensation pressure, as is discussed in the literature [62]. 


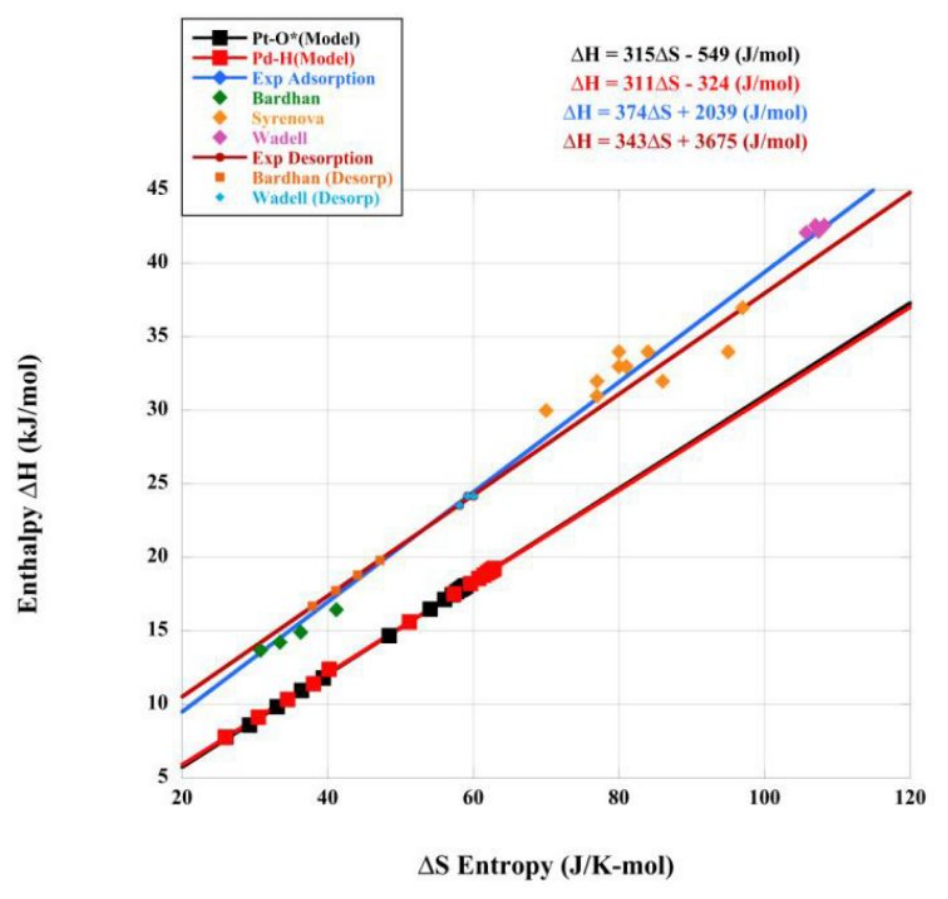

Figure 8: Linear data with the compensation temperature of enthalpy and entropy. The data from Bardhan [56] is for adsorption (green) and desorption (orange), Syrenova [21] is for adsorption, Wadell [58] (pink) is for adsorption and Wadell [55] (light blue) is for desorption. 
The computationally realistic Morse potential model, when used in this coordinationstyle method, has allowed us to gain new insights into the catalytic thermodynamics of some important systems. We recognize that we have made some simplifying assumptions in our calculations, but this has enabled us to model complicated physical and chemical reactions. This method can be done with reasonable computational efficiency, with modest time requirements, and yields surprisingly new and plausible conclusions. We hope that these results will be confirmed by other authors.

\section{Conclusion}

The letter presented a general procedure for determining the catalytic thermodynamics of adsorbates on nanocluster surfaces. In particular, we have derived thermodynamic functions, as entropy, total energy and free energy, of platinum nanoclusters with oxygen adsorbates, using the Morse potential. These functions show linear behavior with adatom coverage. While experimental measurements of the entropy of $\mathrm{Pt}(111)-\mathrm{O}_{2}$, are higher than what we calculate, these methods produce similar results of the entropy compared to other theoretical (CTST) methods. We recognize that the experimental value may be adjusted lower, as the enthalpy has been. The enthalpy of large diameter Pt-O* clusters is close the value found by DFT on bulk Pt-O* at full coverage. The experimental data for $\mathrm{Pd}-\mathrm{H}_{2}$ has quite a bit of scatter, but the average is near both our model for entropy and enthalpy for $\mathrm{Pd}-\mathrm{H}$. Considering that the experiments involve some adsorption of hydrogen into the nanoclusters, our model can be considered a reasonable fit. Finally, a plot of the enthalpy versus entropy gives a linear fit for both the experimental data and our modeled results.

\section{Acknowledgments}

We made use the MATLAB file, Cluster Generator, which can be found in Mathworks File Exchange Central. We thank G.L. Gürtler and E.I. Altman for reviewing the manuscript.

\section{References}

[1] K. Maruyama, F. Nori, V. Vedral, Rev. Mod. Phys. 81 (2009) 1-23.

[2] A. Berut, et al, Nature 483 (2012) 187-189.

[3] S. Toyabe, et al, Nature Physics 6 (2010) 988-992.

[4] J. Koski, V. Maisi, T. Sagawa, J.P. Pekola, Phys. Rev. Lett. 113 (2014) 030601.

[5] J.M.R. Parrondo, J.M. Horowitz, T. Sagawa, Nature Physics 11(2) (2015) 131-139.

[6] J. Goold, M. Huber, A. Riera, L.del Rio, P. Skrzypczyk, J. Phys. A: Math. Theor. 49(14) (2016) 143001.

[7] M. Horodecki, J. Oppenheim, Nature Comm. 4 (2013) 2059.

[8] C.E. Shannon, Bell System Technical Journal 27 (1948) 379-423. 
[9] E.T. Jaynes, Phys. Rev. 106(4) (1957) 620-630

[10] E.T. Jaynes, Phys. Rev. 108(2) (1957) 171-190

[11] E. Estrada, N. Hatano, Chem. Phys. Lett. 439 (2007) 247-251

[12] P.M. Morse, Phys. Rev. 34 (1929) 57-64

[13] L.A. Girifalco, V.G. Weizer, Phys. Rev. 114(3) (1959) 687-690

[14] D.W. Bassett, P.R. Webber, Surface Science 70, (1978) 520-531

[15] J. Rogan, A. Varas, J.A. Valdivia, M. Kiwi, J. Comput. Chem. 34, (2013) 2548-2556.

[16] D. Parodi, R. Ferrando, Phys. Lett. A 367, (2007) 215-219.

[17] F. Calvo, E. Yurtsever, Comput. Theor. Chem. 1021, (2013) 7-15.

[18] Z. Wen, Y.F. Zhu, Q. Jiang, Mater. Chem. Phys. 145 (2014) 51-55

[19] R.A. Johnson, J. Phys F: Metal Phys. 3, (1973) 295-321.

[20] Y. Kang, J.B. Pyo, X. Ye, R.E. Diaz, T.R. Gordon, E.A. Stach, C.B. Murray, ACS Nano 7(1) (2013) 645-653

[21] S. Syrenova, et al, Nature Materials 14(12), (2015) 1236-1244.

[22] F.D. Manchester, A. San-Martin, J.M. Pitre, J. Phase Equilib. 15, (1994) 62-83.

[23] R.J. Behm, K. Chrstmann, G. Ertl, Surface Science 99, (1980) 320-340.

[24] H. Conrad, G. Ertl, E.E. Latta, Surface Science 41, (1974) 435-446.

[25] S. Wilke, D. Hennig, R. Lober, M. Methfessel, M. Scheffler, Surface Science 307-309, (1994) $76-81$.

[26] J.F. Paul, P. Sautet, Phys. Rev. B. 53, (1996) 8015-8027.

[27] W. Niu, L. Zhang, G. Xu, ACS Nano 4(4), (2010) 1987-1996.

[28] E.M. Larsson, C. Langhammer, I. Zoric, B. Kasemo, Science 326, (2009) 1091-1094.

[29] A.S. Mikheykin, V.P. Dmitriev, S.V. Chagovets, A.B. Kuriganova, N.V. Smirnova, I.N. Leontyev, Appl. Phys. Lett. 101, (2012) 173111.

[30] C. Kittel, Introduction to Solid State Physics, Eighth Edition, John Wiley \& Sons, Inc, Hoboken, NJ, 2005.

[31] W. Martienssen and H. Warlimont, Springer Handbook of Condensed Matter and Materials Data Springer: Berlin, 2005.

[32] Q. Jiang, H.M. Lu, Surface Science Reports 63, (2008) 427-454

[33] M.A. Shandiz, J. Phys.: Condens. Matter 20 (2008) 325237

[34] E. Roduner, Chem. Soc. Rev. 35, (2006) 583-592.

[35] E. Shustorovich, J. Am. Chem. Soc., 106, (1984) 6479-6481.

[36] K. Wettergren, A. Hellman, F. Calvalca, V.P. Zhdanov, C. Langhammer, Nano Letters 15, (2015) 574-580.

[37] F. Calle-Vallejo, J.I. Martinez, J.M. Garcia-Lastra, P. Sautet, D. Loffreda, Angew. Chem. Int. Ed. 53 (2014) 8316-8319

[38] F. Calle-Vallejo, J. Tymoczko, V. Colic, Q.H. Vu, M.D. Pohl, K. Morganstern, D. Lof- 
freda, P. Sautet, W. Schuhmann, A.S. Bandarenka, Science 350 (2015) 185-189

[39] W.H. Weinberg, J. Vac. Sci. Technol. 10(1), (1973) 89-94.

[40] J. Han, W. Hu, H. Deng, Surf. Interface Anal. 41, (2009) 590-594.

[41] H.M Lu and Q. Jiang, J. Phys. Chem. B 108 (2004) 5617-5619.

[42] J.L. Gland, Surface Science 93, (1980), 487-514.

[43] L. Li, A.H. Larsen, N. A. Romero, et al, J. Phys. Chem. Lett. 4, (2013) 222-226.

[44] S.H. Sohrab, Int. J. Mechanics 8, (2014) 73-84.

[45] C. Langhammer, E.M Larsson, B. Kasemo, I. Zoric, Nano Lett. 10, (2010) 3529-3538.

[46] Y. Li, W. Qi, B. Huang, M. Wang, J. Phys. Chem. C 116, (2012) 26013-26018.

[47] G.N Vayssilov et al, Nature Mater. 10, (2011) 310-315.

[48] N.I. Jaeger, A.L. Jourdan, G. Schulz-Ekloff, J. Chem. Soc. Faraday Trans. 87(8), (1991) 1251-1257.

[49] G. Kneringer, F.P. Netzer, Surf. Sci. 49, (1975) 125-142.

[50] L.K. Ono, B. Yuan, H. Heinrich, B.R. Cuenya, J. Phys. Chem. C 114, (2010) 2211922133.

[51] L.K. Ono, J.R. Croy, H. Heinrich, B.R. Cuenya, J. Phys. Chem. C 115, (2011) 1685616866.

[52] C.J. Pursell, H Hartshorn, T. Ward, B.D. Chandler, F. Boccuzzi, J. Phys. Chem C 115, (2011) 23880-23892.

[53] C.J. Pursell, B.D. Chandler, M. Manzoli, F. Boccuzzi, J. Phys. Chem C 116, (2012) 11117-11125.

[54] E.M Karp, C.T. Campbell, F. Studt, F. Abild-Pederson, J.K. Norskov J. Phys. Chem C 116, (2012) 26772-25776.

[55] C. Wadell, PhD Thesis, Chalmers Univ. Technolody, Sweden, (2015).

[56] R. Bardhan et al, Nature Mater. 12, (2013) 905-912.

[57] R. Lasser, K.H. Klatt, Phys. Rev. B 28, (1983) 748-758.

[58] C. Wadell et al, Chem. Phys. Lett. 603, (2014) 75-81.

[59] C. Sachs, et al, Phys. Rev. B 64, (2001) 075408.

[60] J. O’M. Bockris, R. Abdu, Electroanal. Chem. 448, (1998) 189-204.

[61] V. Fiorin, D. Borthwick, D.A. King, Surf. Sci. 603, (2009) 1360-1364.

[62] R. Griessen, N. Strohfeldt, H. Giessen, Nature Mater. 15, (2016) 311-317. 